\title{
First test of cold edgeless silicon microstrip detectors
}

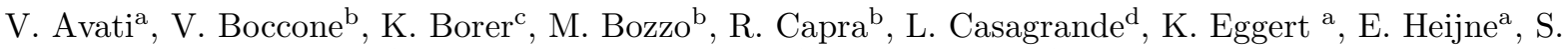

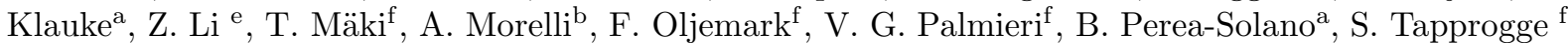 \\ ${ }^{\mathrm{a}} \mathrm{CERN}$, Geneva, Switzerland \\ ${ }^{\mathrm{b}}$ INFN Sez. di Genova and Universitá di Genova, Genoa (Italy) \\ ${ }^{\mathrm{c}}$ Laboratorium fuer Hochenergiephysik, Universitaet Bern, Bern, Switzerland \\ dINFN Sez. di Roma 2 and Universitá di Roma 2, Rome (Italy) \\ eBrookhaven National Laboratory, Upton NY (USA) \\ ${ }^{\mathrm{f}}$ Helsinki Institute of Physics, Helsinki (Finland)
}

\begin{abstract}
Silicon microstrip detectors will provide the forward tracking in the TOTEM experiment at the LHC. To allow tracking closest to the beam $(\approx 1 \mathrm{~mm})$ these detectors should be sensitive up to their physical edge (i.e. edgeless). Edgeless (without guard rings) microstrip planar detectors can be operated at cryogenic temperatures (about $130^{\circ} \mathrm{K}$ ) where leakage currents due to the active edge are drastically reduced. A silicon microstrip prototype, cut perpendicular to the strips, has been tested with a pion beam at CERN to study its efficiency close to the edge by using reference tracks from a simple silicon telescope. Results indicate that the detector measures tracks with good efficiency up to the physical edge of the silicon.
\end{abstract}

\section{Introduction}

The TOTEM experiment[1] at the CERN LHC needs detectors to track with high efficiency elastically scattered protons very close to the circulating beams. The accuracy of the measurement of the total cross section critically depends on the smallest momentum transfer observable. This requires an edgeless detector, with very high and stable efficiency close to the mechanical border on the side facing the beam, a good spatial resolution of the order of $20 \mu \mathrm{m}$, and a fair resistance to radiation damage. Silicon microstrip detectors are the natural candidate. However they must be operated without guard rings $^{1}$ minimizing surface leakage current. TOTEM's detectors with a typical size of $\left(\approx 3 \times 4 \mathrm{~cm}^{2}\right)$ will be inserted in special sections of the LHC vacuum chamber (Roman Pots).

Studies from RD39/NA60 on the operation of

${ }^{1}$ present technology guard rings have dimensions of $\approx$ $500 \mu m$ silicon microstrip detectors cooled at cryogenic temperature have hinted[2][3] that a normal silicon detector would show only a small, if any, increase in leakage current when operated without a guard rings structure.

Detector without guard rings had never been operated before. Considering the importance of such novel idea for the TOTEM experiment we present here a "proof of principle" measurement performed with a very simple and minimal setup.

\section{Description of the measurement setup}

The setup (fig.1)consist of a tracking hodoscope with 4 microstrip silicon detectors (two with vertical and two with horizontal strips) that define the particle beam with a precision of $20 \mu \mathrm{m}$ in the middle plane where the cut microstrip detector is placed. Exposing this set-up to a pion beam allows a precise efficiency study of the edge of the detector.

The measuring stations are equipped with mi- 


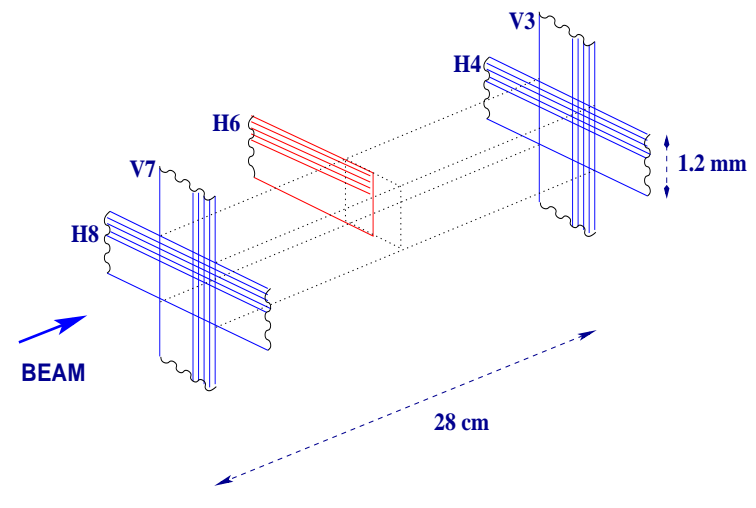

Figure 1. The setup used for the test.

crostrip silicon detectors of the same kind as the ones employed for the beam telescope of experiment NA60[4]. Two detectors are mounted back to back with the strips perpendicular to each other on a glass-epoxy printed circuit board. NA60 $\mathrm{Al} / \mathrm{p}^{+} / \mathrm{n} / \mathrm{n}^{+} / \mathrm{Al} 400 \mu \mathrm{m}$ thick microstrip detectors have 24 strips of $50 \mu \mathrm{m}$ pitch and 4 strips $500 \mu \mathrm{m}$ wide, all surrounded by a single guard ring. The wide lateral strips were not used for this test and thus the final sensitive area was $1.2 \times 1.2 \mathrm{~mm}^{2}$. The detectors were produced at the BNL silicon facility and some were cut perpendicular to the strips implant by first scribing them from the backplane with a laser beam and then by bending them to crack. With this method a clean edge is produced at the strip side (fig2). The detector was not submitted to any other treatment after this operation. However, treatments of the cut edge seems to eliminate surface defects[5] to further reduce the leakage current. In order to cool the silicon detectors liquid nitrogen is fed to capillaries soldered on a 6 layers PCB which was designed to minimize heat conduction between the detector and the flange at room temperature. The three detector stations are flushed in parallel with flow equalization at the gas outputs. The temperature is monitored on the board close to the detector and was set to $110^{\circ} \mathrm{K}$ by controlling the amount of LN flow and the power dissipated trough a heater on the
PCB. Since vacuum is needed for thermal insulation the detectors were mounted on the flange of a cryostat. All detectors were biased at $45 \mathrm{~V}$ showing a leakage current of less than $15 n A$ at the operating temperature of $110^{\circ} \mathrm{K}$.

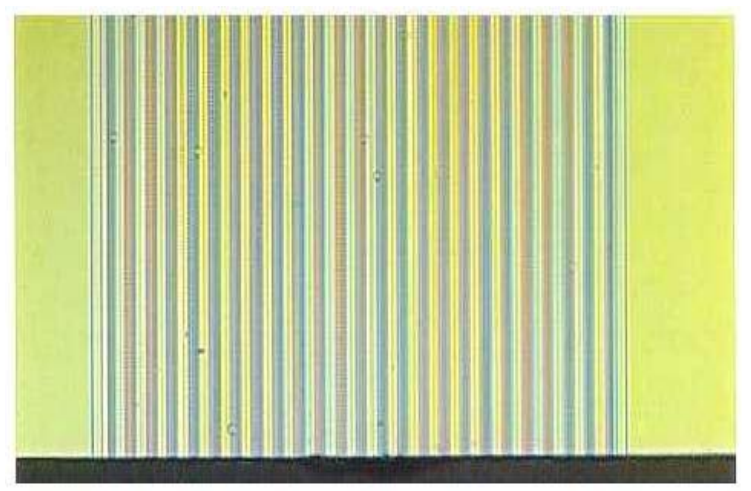

Figure 2. Enlarged picture of the edge of the microstrip detector. The scale is given by the pitch of the 24 strips which is $50 \mu \mathrm{m}$.

The signals from the preamplifier[6] mounted on the PCB near the detector were sent via an adapter board to a shaping amplifier outside the cryostat, then sampled and multiplexed in a FADC for recording. We did not make a special effort to optimize the noise of the system. However, it was possible to obtain data of sufficient quality to perform the measurement.

The relative positions of the silicon detectors were carefully measured in the $\mathrm{lab}^{2}$ after the measurement. The results of these measurements have been taken into account during the data analysis.

\section{Results}

The detectors were exposed to a $120 \mathrm{GeV}$ pion beam with an intensity of $10^{6}$ particles per spill

\footnotetext{
2 the measurement conditions were recreated in the lab to measure possible deformations of the flange due to the vacuum.
} 


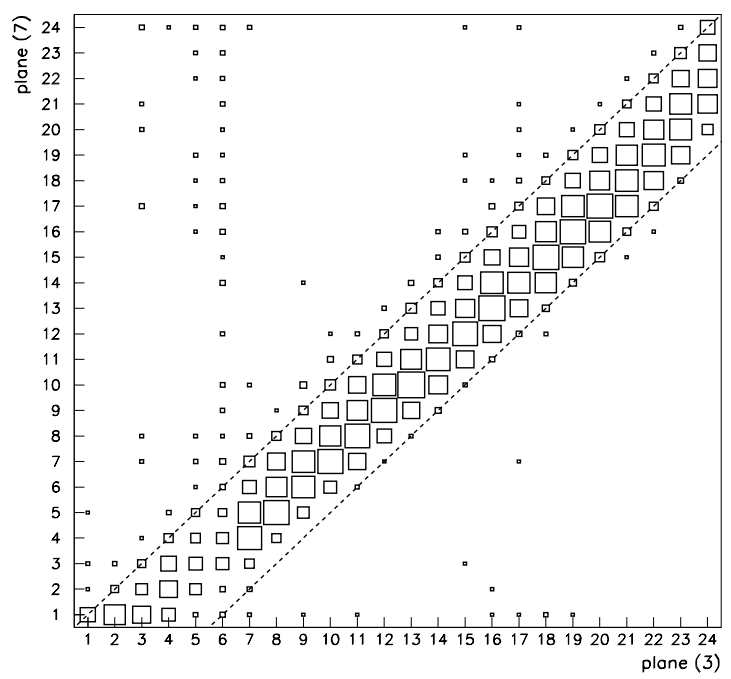

Figure 3. The correlation between the strips hit in the vertical plane (the dotted line indicates the cut applied to the data).

length of $2.2 \mathrm{~s}$. The trigger was provided by a coincidence of a large and a small $3 \mathrm{~mm}$ diameter scintillator counter. The small counter covered a fair fraction of the narrow beam profile and was mounted $5 \mathrm{~m}$ downstream on a motorized stage to select particles aligned with the set-up.

To define the tracks with the hodoscope a pulse-height cut for each strip of detectors V3,V7 and H8 (see Fig.1) was applied at 3 sigma above the mean pedestal value. For the cut detector H6 a lower 2 sigma cut guaranteed a high detection efficiency at the expense of a slightly increased background. The correlation between the vertical strips in V3 and V7 and the horizontal strips in $\mathrm{H} 8$ and $\mathrm{H} 6$ is plotted in Fig.3 and 4.

The relative displacements of the strips in the detectors are in agreement with the measurement of the metrology which also confirms that the beam direction was perpendicular to the detector planes. To clean the data, tracks were accepted if they were in both projections within the dotted lines. This cut corresponds to an angular beam spread of less than 0.3 mrad.

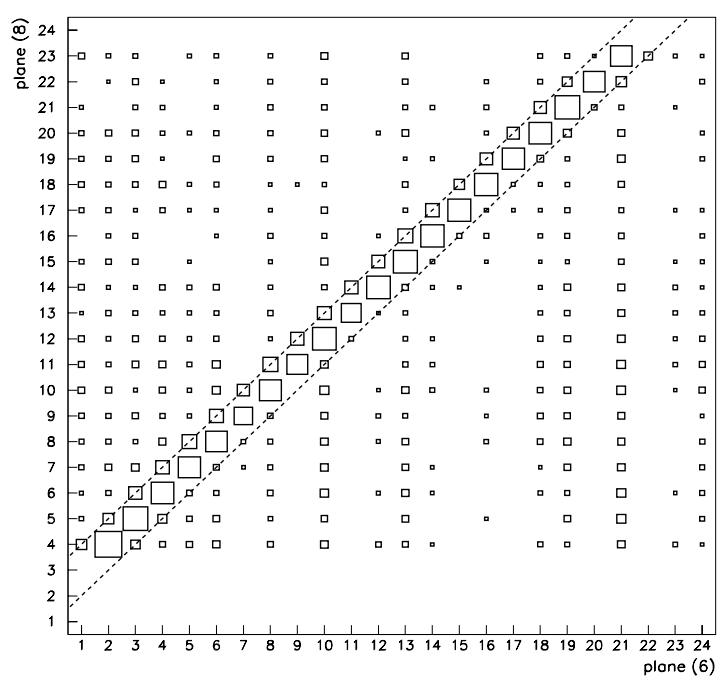

Figure 4. The correlation between the strips hit in the horizontal plane (the dotted line indicates the cut applied to the data).

Fig. 5 shows the same vertical correlation plot as Fig.3, but now for the case where the cut silicon detector had a hit on the strips in a narrow road of 3 strips. The sharp cut in the beam profile is clearly visible with only a few background hits. From the metrology we know within a precision of $20 \mu \mathrm{m}$ the position of the mechanical edge of the cut detector with respect to the vertical strips of the detectors V3 and V7. In Fig. 6 the distance of the tracks to the mechanical edge of the cut detector is plotted in $25 \mu \mathrm{m}$ bins for the two cases: no requirement on the cut detector and a hit in the cut detector within the road indicated in Fig.3. The ratio of the two curves (shown in Fig.7 ) is the efficiency of H6. The steep rise of the efficiency at the mechanical edge of the cut detector is compatible with a full detector efficiency up to the edge. However, given the uncertainties in the metrology measurement, we claim more conservatively that the cut detector is fully efficient within a distance $0+25 \mu \mathrm{m}$ of the edge.

We want to thank the CERN Cryolab for their invaluable help in cooling the detectors. 


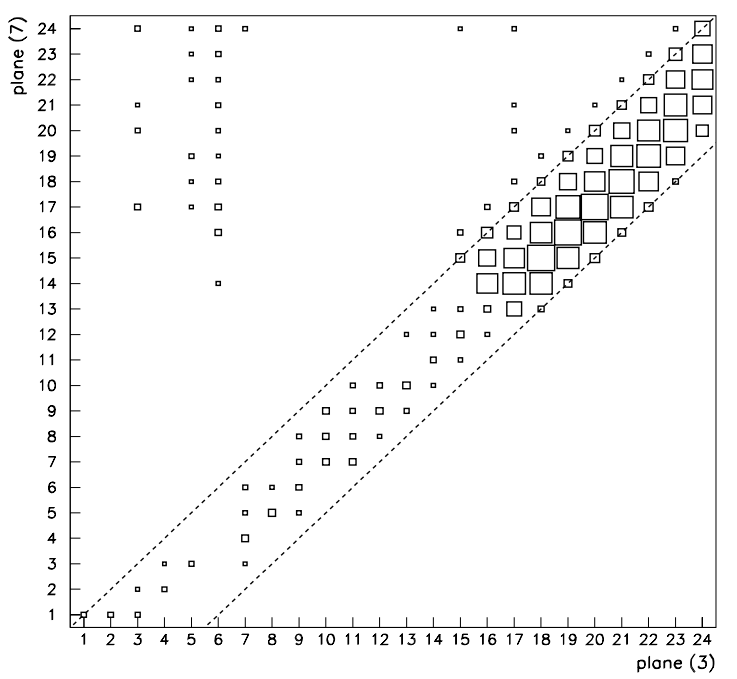

Figure 5. The correlation between the strips hit in the horizontal plane when a hit is recorded in the cut silicon (the dotted line indicates the cut applied to the data).

\section{REFERENCES}

1. TOTEM Collaboration, W. Kienzle et al., Technical proposal, CERN/LHCC 99-7 LHCC/P5, 1999.

2. K.Borer et al., NIM A 440 (2000) 5. S.Grohman et al., IX Blois Work. on El. and Diff. Scatt., Pruhonice, Czech Republic, (2001), 363.

3. W.H. Bell et al., Proceedings $8^{t} h$ Elba Meet. 2000

4. L.Casagrande et al., NIM A (2002) 325-329.

5. Z. Li et al, IEEE NSS Proc., San Diego, Nov. 2001

6. G. Anelli et al., presented at ELMAU 2002, acc. for pub. in NIM A

7. "RD39 Status Report", CERN/LHCC 2002004, 09 January 2002

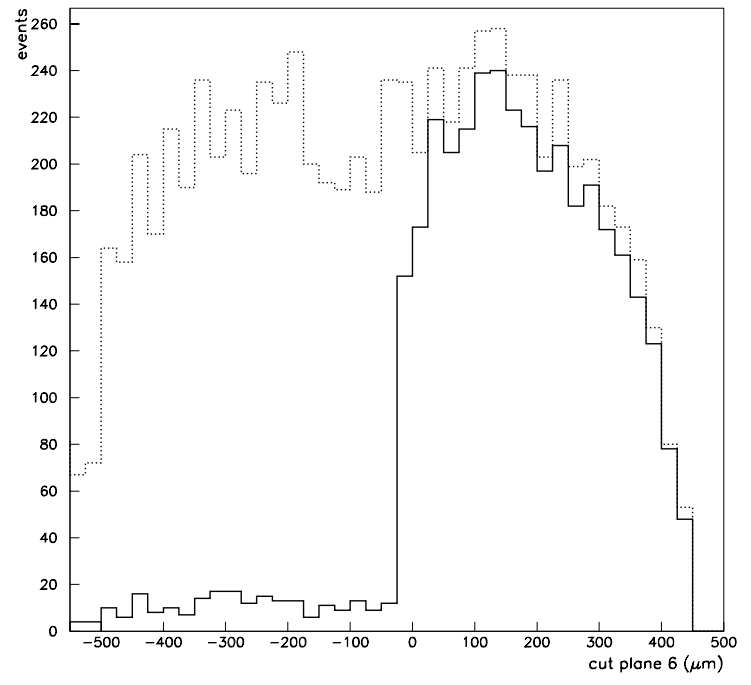

Figure 6. The projection of the plot of the correlation between the strips hit in the horizontal plane with (solid line) and without (dotted line) a hit in the cut silicon detector.

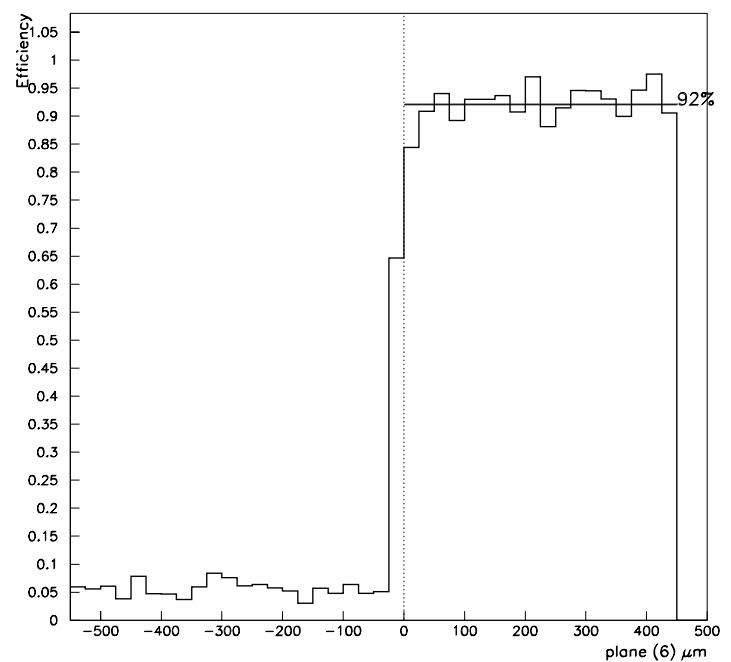

Figure 7. the plot of the efficiency of the cut silicon detector. 